\title{
Novos Biomateriais: Híbridos Orgânico-Inorgânicos Bioativos
}

\author{
Ana Paula V. Pereira, Wander L. Vasconcelos e Rodrigo L. Oréfice
}

Resumo: Materiais bioativos têm a capacidade de interagir com tecidos naturais, provocando reações que favoreçam o desenvolvimento de processos como: fixação de implantes, biocolonização, regeneração de tecidos anfitriões ou biodegradação do material. Grande parte dos materiais bioativos são cerâmicas. $\mathrm{O}$ objetivo deste trabalho envolve o desenvolvimento de novos biomateriais que apresentem níveis controláveis de reatividade a partir da combinação a nível nanométrico ou molecular de fases poliméricas e inorgânicas. Neste trabalho foram produzidos híbridos combinando álcool polivinílico (PVA) e tetraetil ortossilicato ou tetrametil ortossilicato dopados com sais de cálcio e alcóxido de fósforo. A caracterização do material produzido foi feita utilizando-se as seguintes técnicas: teste de inchamento por solvente, espectroscopia de infravermelho, microscopia eletrônica e análise térmica (DSC e TGA). O grau de reatividade foi determinado a partir da inserção das amostras em soluções aquosas por diferentes períodos de tempo. A partir do procedimento desenvolvido foram produzidos híbridos, na forma de filmes transparentes e flexíveis, de PVA-silicatos de cálcio e fósforo com composição variando de 25 a $100 \% \mathrm{em}$ volume de PVA no híbrido e 40 a $80 \%$ molar de sílica na fase inorgânica. Os testes de imersão em solução aquosa demonstraram que os híbridos produzidos podem exibir reatividade controlável, variando de elevada degradabilidade até comportamento tipo gel, determinado através da avaliação da cinética de lixiviação e precipitação dos íons cálcio e fosfato. Os resultados mostraram ainda que o grau de reatividade dos materiais pode ser controlado através da manipulação de fatores estruturais como a densidade de ligações cruzadas, proporção das fases, densidade e composição da fase inorgânica, entre outros.

Palavras-chave: Híbridos, materiais bioativos, biomateriais, álcool polivinílico, biopolímeros.

\section{Introdução}

O tema central do trabalho abrange o estudo e desenvolvimento de nanocompósitos envolvendo polímeros e cerâmicas para fins biomédicos. Em termos de biomateriais, uma análise da estrutura dos tecidos que constituem o corpo humano permite mostrar que os mesmos são tipicamente compósitos. Assim sendo, tais materiais são formados por dois ou mais componentes que, em conjunto, são responsáveis pelas propriedades finais do tecido. A partir de tal consideração, sugere-se que a substituição de tecidos vivos por materiais sintéticos pode estar ligada ao desenvolvimento de compósitos especiais capazes de reproduzir as propriedades dos tecidos humanos.

A escolha de um material para ser usado como biomaterial passa necessariamente pela análise de

Ana Paula V. Pereira, Wander L. Vasconcelos, Rodrigo L. Oréfice, LEPCom, Laboratório de Engenharia de Polímeros e Compósitos, Departamento de Engenharia Metalúrgica e de Materiais, Universidade Federal de Minas Gerais, CEP: 30160-030, Belo Horizonte, MG. E-mail: rorefice@demet.ufmg.br 
um conjunto de requisitos que devem ser encontrados. O efeito do ambiente orgânico no material (corrosão, degradação) e o efeito do material no organismo são fenômenos que devem ser estudados com extremo cuidado, pois a eles está associado a chamada "biocompatibilidade". Dentre esses dois últimos aspectos, a interação dos tecidos vivos com o biomaterial, associada com o tipo de resposta do organismo à presença do material, é o ponto mais desafiador no desenvolvimento de biomateriais. Os tipos de interação entre tecidoimplante são fundamentalmente dependentes do tipo de material e podem ser reunidos nos seguintes grupos ${ }^{[1]}$ : tóxica, não-tóxica (muitas vezes chamada de bioinerte), bioativa e biodegradável. Ultimamente, o desenvolvimento de materiais considerados bioativos e biodegradáveis vêm sendo enfatizado já que, além de substituir tecidos traumatizados, estes materiais também podem propiciar a recuperação do tecido danificado através da atuação em metabolismos intra e extracelulares responsáveis pela reprodução celular e propagação dos tecidos em crescimento.

Em termos de materiais biodegradáveis, um dos grandes objetivos de bioengenheiros e cientistas é a produção de biomateriais capazes de substituir tecidos danificados por um certo período de tempo durante o qual o processo de reparo natural da área afetada estaria sendo promovido. O material ideal para esta função, além de biodegradável, estimularia o regeneração do tecido matriz e teria uma cinética de degradação das propriedades mecânica compatível com a cinética de reparo do tecido. Dessa forma o novo tecido iria progressivamente substituindo o implante nas funções requeridas.

O sistema álcool polivinílico-vidro bioativo pode levar a preparação de nanocompósitos com bioatividade (biodegradabilidade) controlada. Álcool polivinílico é um polímero solúvel em água (e consequentemente no ambiente fisiológico) e está sendo cogitado para ser usado como sangue artificial e matriz para sistema de fornecimento de substâncias químicas no corpo (drug delivery system). Ao mesmo tempo, vidros bioativos podem ser biodegradáveis quando possuidores de alto teor de agentes modificadores de reticulado (cálcio e fósforo) e preparados em submicrométrcas dimensões ${ }^{[1]}$.

A produção de materiais com fases inorgânicas submicroscópicas inseridas em uma matriz polimérica pode ser alcançada através da síntese de nanocompósitos, também chamados de inorgânico-orgânicos híbridos. A possibilidade de combinação de entidades orgânicas e inorgânicas em níveis nanométricos e moleculares em orgânico-inorgânico híbridos capacita a abertura de uma série de novas oportunidades para compósitos com controláveis propriedades e estrutura ${ }^{[2-5]}$. Tal tipo de organização estrutural em materiais sintéticos se assemelha em grande extensão, a estrutura presente em alguns tecidos vivos, como o osso, onde o constituinte inorgânico (hidroxiapatita carbonatada) e entidades orgânicas, como colágeno, se interagem em diminuta escala, formando nanofases ligadas entre si por ligações químicas de primeira ordem $^{[6]}$. Assim, a ciência e tecnologia relacionada com híbridos inorgânico-orgânicos potencialmente pode ser aplicada à preparação de uma nova classe de materiais semelhante estruturalmente a tecidos vivos (nanocompósitos polímero-cerâmica) para aplicações médicas e odontológicas.

Dessa forma, os compósitos híbridos propostos neste trabalho tem o potencial de atender esta demanda em relação a materiais biodegradáveis com degradação química e mecânica controláveis. Neste caso, as velocidades de degradação podem ser controladas através da manipulação de variáveis como fração volumétrica do componente inorgânico, tipo de interação entre componentes, tamanho e distribuição das fases. Além disso, vidros bioativos são comprovadamente dotados da capacidade de aumentar as velocidades de reparo dos tecidos ${ }^{[1]}$. Assim, o uso destes materiais nos compósitos biodegradáveis pode ser benéfica em termos de acelerar a reconstituição de tecidos danificados.

Neste trabalho pretende-se desenvolver compósitos híbridos (híbridos inorgânico-orgânico) bioativos cuja bioatividade, ou seja, grau de interação do material com os tecidos vivos, seja controlada através da manipulação de características químicas e estruturais dos materiais.

\section{Experimental}

Os seguintes reagentes foram usados durante a síntese dos materiais: água deionizada, álcool polivinílico, solventes (THF, benzeno, tolueno, etanol), tetrametoxissilano (TMOS), nitrato de cálcio e fosfato de trietila. 
Híbridos foram preparados via dissolução do álcool polivinílico em água e adição de TMOS à solução. Em seguida, quantidades pré-determinadas de nitrato de cálcio e fosfato de trietila foram introduzidos na solução. As soluções finais foram vazadas sobre lâminas de vidro. A gelação ocorreu em média em três horas à temperatura ambiente.

A proporção relativa das fases dos compósitos álcool polivinílico/sílicato foi alterada através da variação da quantidade de TMOS adicionada ao polímero. A composição da fase inorgânica também foi variada a partir da introdução de diferentes quantidades de reagentes. As composições dos híbridos preparados estão mostradas na Tabela 1 .

Filmes produzidos foram tratados termicamente à $60^{\circ} \mathrm{C}$ por 24 e 50 horas.

A densidade de ligações cruzadas foi determinada pela técnica de inchamento, onde os materiais são submetidos à solventes e a fração de solvente retida no reticulado é usada na teoria de Flory para determinação da fração de ligações cruzadas. Neste trabalho, os materiais produzidos foram inseridos por 1 hora num frasco com água deionizada na temperatura de $25^{\circ} \mathrm{C}$. As massas antes $\left(\mathrm{m}_{0}\right)$ e após $\mathrm{o}$ teste $(\mathrm{m})$ foram medidas e usadas na determinação do grau de inchamento, Q (1):

$$
\mathrm{Q}=\left(\mathrm{m}-\mathrm{m}_{0}\right) /\left(\mathrm{m}_{0} \rho\right)
$$

Onde: $\rho=$ densidade do solvente $\left(\rho=1,0 \mathrm{~g} / \mathrm{cm}^{3}\right)$.

Microanálise EDS acoplada à um Microscópio Eletrônico de Varredura (JEOL JSM 5410) foi usada para avaliação semi-quantitativa da composição química das amostras. As amostras foram metalizadas com liga Au-Pd para análise.

A espectroscopia na região do infravermelho por transformada de Fourier (FTIR) foi usada na caracterização química dos materiais (PerkinElmer, Paragon 1000). Utilizou-se a técnica de reflectância total atenuada (ATR) na avaliação estrutural dos filmes. Neste caso, os filmes produzidos foram pressionados contra a superfície de um cristal de $\mathrm{ZnSe}$. Foram obtidos espectros entre 4000 e $700 \mathrm{~cm}^{-1}$ durante 64 varreduras.

\section{Resultados e Discussão}

A Figura 1 mostra os espectros EDS para nanocompósitos PVA-silicatos de cálcio e fósforos para amostras com diferentes proporções de fração inorgânica e porcentagens de óxidos (ou hidróxidos) de cálcio e fósforo. As razões entre as intensidades dos picos relativos ao silício ( $\mathrm{Si}$ ) e carbono (C) podem ser usadas para demonstrar a capacidade do método de preparação desenvolvido em obter composições projetadas dentro de um espectro de possibilidades relacionadas com as quantidades relativas das fases (inorgânica versus orgânica). Assim, o espectro da amostra C3 apresenta uma proporção maior de fase inorgânica do que a amostra A3. As razões entre intensidades dos picos entre $\mathrm{Si}$ e $\mathrm{Ca}$ permite constatar a habilidade do método de síntese desenvolvido na preparação de fases inorgânicas

Tabela 1. Híbridos multicomponentes PVA-silicatos de cálcio e fósforo.

\begin{tabular}{ccccc}
\hline Amostra & $\begin{array}{c}\text { Proporção das fases (em peso)* } \\
\text { Fase Inorgânica/PVA }\end{array}$ & \multicolumn{2}{c}{ Composição da fase inorgânica (\% em peso)** } \\
\hline & & $\mathrm{SiO}_{2}$ & $\mathrm{CaO}$ & $\mathrm{P}_{2} \mathrm{O}_{5}$ \\
$\mathrm{~A} 1$ & 0,5 & 60 & 36 & 4 \\
$\mathrm{~A} 2$ & 0,5 & 50 & 46 & 4 \\
$\mathrm{~A} 3$ & 0,5 & 80 & 16 & 4 \\
$\mathrm{~B} 1$ & 60 & 36 & 4 \\
$\mathrm{~B} 2$ & 50 & 46 & 4 \\
$\mathrm{~B} 3$ & 1,0 & 80 & 16 & 4 \\
$\mathrm{C} 1$ & 1,0 & 60 & 36 & 4 \\
$\mathrm{C} 2$ & 1,0 & 50 & 46 & 4 \\
$\mathrm{C} 3$ & 1,5 & 80 & 16 & 4 \\
\hline
\end{tabular}

* Proporção entre os reagentes iniciais (alcóxidos/PVA);

** Calculada assumindo total hidrólise dos precursores. 


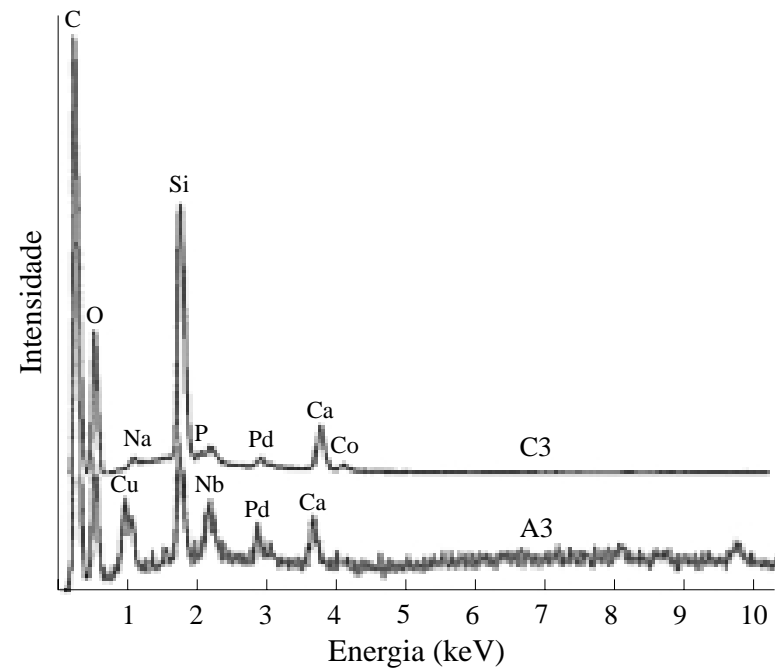

Figura 1. Análises EDS de amostras de A3 e C3 (Tabela 1).

com composições previamente estipuladas. Além disso, a presença de valores próximos para as razões entre picos $\mathrm{Si} / \mathrm{Ca}$, observadas nos espectros da Figura 1, certifica a produção de amostras diferentes (A3 e C3) com fases inorgânicas com composições similares.

A Espectroscopia de Infravermelho (FTIR) foi utilizada neste trabalho para prover informações relacionadas com a o efeito da adição dos precursores de cálcio e fósforo na estrutura da fase inorgânica. Os espectros da Figura 2 dizem respeito à três amostras com mesma proporção entre fases (inorgânica versus polimérica), mas com fases inorgânicas possuindo diferentes composições $(\mathrm{C} 1, \mathrm{C} 2, \mathrm{C} 3)$. Todos os três espectros da Figura 2 apresentam uma série de similaridades oriundas justamente do fato de que são produzidos com mesma fração de fase inorgânica. Assim sendo, os espectros de FTIR apresentam basicamente picos relacionados com o álcool polivinílico entre os quais se nota a presença dos picos relativos à fase inorgânica. Tais picos da fase inorgânica se mostram mais evidentes nas regiões entre 1100-1000, 950-900 e 800-700 $\mathrm{cm}^{-1}$, onde se caracterizam respectivamente as regiões de absorção das ligações Si-O-Si (modo de estiramento assimétrico), ligações $\mathrm{Si}-\mathrm{OH}$ (modo de estiramento) e ligações Si-O-Si (modo de estiramento simétrico). O espectro de FTIR na parte superior da Figura 2 foi produzido a partir de uma subtração espectral onde a contribuição referente ao PVA puro foi subtraída dos espectros 2-b e 2-c. O resultado da subtração evidencia a presença dos picos característicos da sílica, assim como permite uma discussão sobre

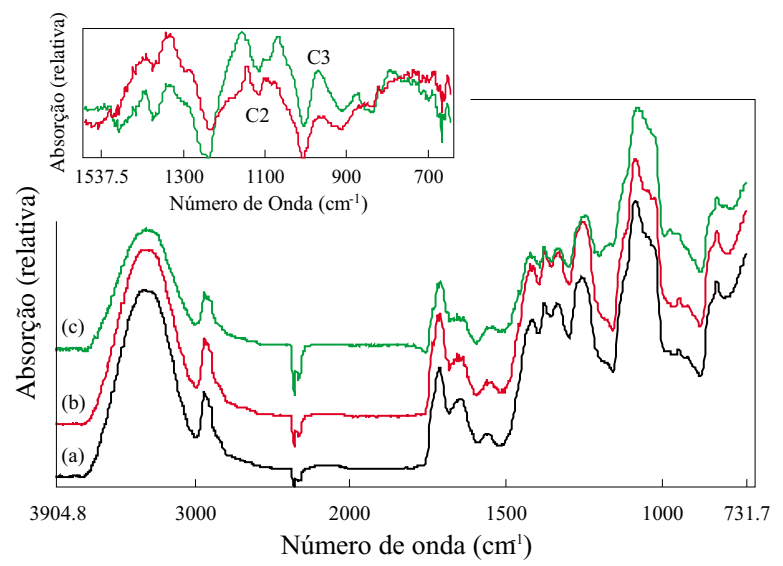

Figura 2. Espectros de FTIR: (a) amostra C1; (b) amostra C2; (c) amostra C3 (Tabela 1). Parte superior: resultado de subtração espectral entre o espectro do PVA puro e espectros (b) e (c).

a forma de organização do reticulado tridimensional deste material em respeito à adição de elementos precursores de cálcio e fósforo. Para este fim, a relação entre a intensidade dos picos correspondentes à ligação Si-O-Si $\left(1050 \mathrm{~cm}^{-1}\right)$ e ligação $\mathrm{Si}-\mathrm{OH}$ $\left(950 \mathrm{~cm}^{-1}\right)$ fornece informação sobre a efetividade de introdução dos precursores de cálcio e fósforo no reticulado tridimensional da sílica. A presença de menores razões entre Si-O-Si e SiOH para amostras projetadas a apresentarem maior teor em sílica (amostra C3, espectro 2-c) do que amostras mais ricas em $\mathrm{Ca}$ e $\mathrm{P}$ (A3) demonstra a existência de fases inorgânicas com maiores concentrações de ligações Si-OH para a amostra C3 (Espectro 2-c). Tal resultado comprova a incorporação em maior número de ligações $\mathrm{Ca}-\mathrm{O}$ nos reticulados de sílica da amostra A3, já que este tipo de ligação substitui ligações Si-OH, dando origem a ligações multicomponentes Si-O-Ca. Dessa forma, os resultados de FTIR demonstraram a capacidade do método desenvolvido de síntese em produzir nanocompósitos com fases ricas em silicatos contendo cálcio e fósforo.

Nas Figuras 3 e 4, o grau de inchamento dos híbridos produzidos e tratados termicamente a diferentes temperaturas e tempos é exibido. Um resultado importante mostrado nestas figuras diz respeito à capacidade dos híbridos de se dissolverem (representado nos gráficos por valores de grau de inchamento tendendo a infinito) dependendo de suas composições e estruturas. Assim, híbridos do sistema em estudo podem ser preparados à assumir diferentes comportamentos frente à presença do solvente, variando desde rapidamente 
dissolvíveis (tempos menores que 1 hora) até estáveis com elevados graus de inchamento (hidrogéis). A dissolução dos híbridos multicomponentes produzidos pode ser assim explicada: (1) a estrutura do híbrido obtido apresenta porções poliméricas hidrosolúveis ligadas entre si por nanométricas fases inorgânicas; (2) a introdução de cálcio e fósforo nas fases ricas em sílica quebra o reticulado inorgânico, forçando a produção de fases inorgânicas mais abertas; (3) quando tais híbridos são inseridos em ambiente aquoso ocorre ataque hidrolítico à ligações $\mathrm{Si}-\mathrm{O}-\mathrm{Ca}$ com a quebra dessas ligações; (4) com a dissolução da fase inorgânica, há a liberação das cadeias de PVA para também se dissolverem. Na Figura 3, o efeito da composição da fase inorgânica no grau de inchamento e dissolução dos materiais é mostrado para híbridos com elevada fração de fase inorgânica (amostras: C1, C2 e C3 - Tabela I). Os resultados mostraram que híbridos, quando produzidos e mantidos à $30^{\circ} \mathrm{C}$, têm uma imensa capacidade de absorver água e dissolução. Para se aumentar a estabilidade das amostras, foi feito um tratamento térmico nos híbridos à $60^{\circ} \mathrm{C}$ por 24 horas ou 50 horas. O tratamento térmico se mostrou efetivo em aumentar a estabilidade das amostras, como mostrado nas Figuras 3 e 4 . Amostras tratadas à temperaturas mais elevadas apresentam reticulado inorgânico mais denso devido ao processamento de reações de condensação entre grupos Si-OH não ocorridas durante a produção dos materiais.

Analisando-se as curvas apresentadas na Figura 3, pode-se perceber que o comportamento dos híbridos tratados termicamente durante 24 e 50 horas, com relação à absorção de água, é bem parecido. Para ambos os casos, existe uma porcentagem de cálcio, aproximadamente $36 \%$ molar, na qual a absorção de água (grau de inchamento) é máxima. Para porcentagens maiores ou menores do que esta, há uma queda na absorção. O efeito da composição da fase inorgânica no comportamento dos híbridos frente solvente está mais uma vez relacionada com o tipo de reticulado inorgânico. Pequenas concentrações de cálcio nos híbridos significa redes sílica mais rígidas e tridimensionais. Com o aumento da concentração de cálcio, há uma progressiva substituição de ligações tetraédricas SiO-Si por ligações lineares Ca-O-Si, o que acaba gerando reticulados mais abertos. Para fases

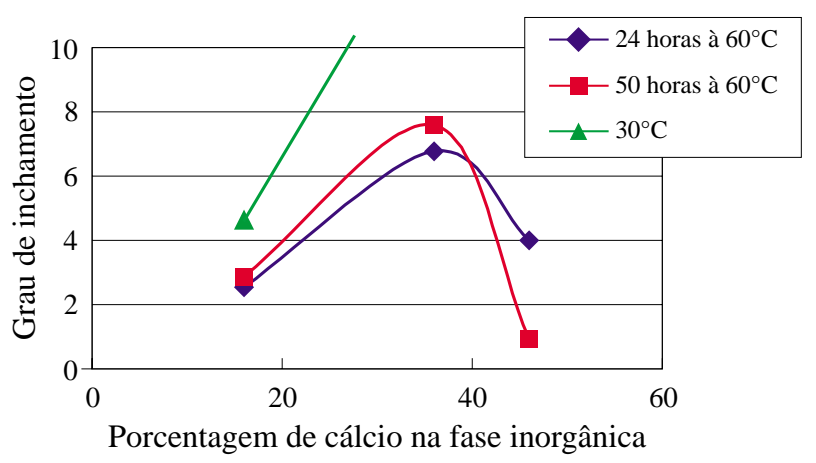

Figura 3. Grau de inchamento para híbridos com fase inorgânica/ PVA $=1,5$ tratados termicamente à $30^{\circ} \mathrm{C}$ e $60^{\circ} \mathrm{C}$ por tempos diferentes.

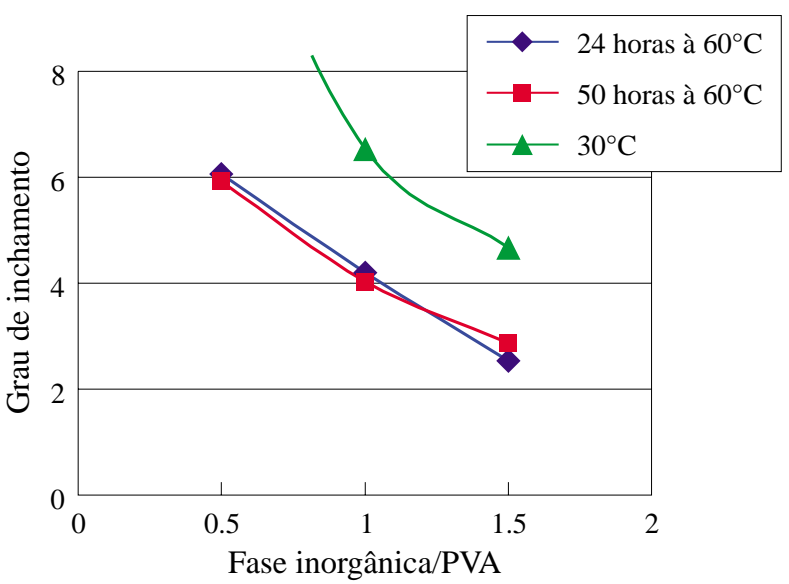

Figura 4. Grau de inchamento em função da variação da proporção entre as fases inorgânica e orgânica para híbridos tratados termicamente à $30^{\circ} \mathrm{C}$ e $60^{\circ} \mathrm{C}$ por tempos diferentes.

inorgânicas com alto teor de cálcio, há formação de regiões ricas em hidróxidos de cálcio que acabam enrijecendo a rede inorgânica.

$\mathrm{Na}$ Figura 4, o efeito da proporção das fases (polimérica versus inorgânica) é examinada para híbridos com fases inorgânicas ricas em sílica (amostras A3, B3 e C3). Fica claro por esta figura que híbridos com maiores frações inorgânicas apresentam maior estabilidade frente ao solvente e menores graus de inchamento. Tal resultado comprova o fato de que as regiões inorgânicas funcionam como pontes ou ligações cruzadas entre as cadeias poliméricas. Dessa forma, mais espessas e rígidas regiões inorgânicas significam maior resistência à deformação originada pelo solvente.

\section{Conclusão}

Neste trabalho, híbridos PVA-silicatos possuindo fases inorgânicas multicomponentes dentro 
do sistema sílica-cálcio-fósforo foram produzidos na forma de filmes transparentes e flexíveis. Tais filmes apresentaram comportamento diferenciado frente ao solvente (água), variando de solúveis até hidrogéis. Este comportamento pôde ser alterado através de variáveis do processo (como temperatura de tratamento térmico) assim como através da composição do sistema. Híbridos tratados à temperaturas mais elevadas apresentaram-se mais estáveis em relação à dissolução. Ao mesmo tempo, híbridos com fases inorgânicas com composições ricas em sílica ou ricas em cálcio se mostraram também mais resistentes à dissolução, possuindo menores graus de inchamento. Híbridos com maiores proporções de fase inorgânica também exibiram menores graus de inchamento.

\section{Agradecimento}

Os autores agradecem à Fundação de Desenvolvimento da Pesquisa (Projeto Fundo FUNDEP) e Conselho Nacional de Desenvolvimento Científico e Tecnológico (CNPq) pelo suporte financeiro à este trabalho.

\section{Referências Bibliográficas}

1. Hench, L. L. - Bioceramics: from concept to clinic. J. Am. Ceram. Soc., 74, n. 7, 1487-510, (1991).

2. Brennan, A. B.; Miller, T. M. - Glasses, organicinorganic hybrids. Kirk-Othmer Encyclopedia of Chemical Technology 4 ed, Volume No. 12, Ed. John Wiley \& Sons, Inc. (1994).

3. Hench, L. L.; Oréfice, R. L. - Sol-Gel Technology, in Encyclopedia Kirk-Othmer of Chemical Technology - $4^{\circ}$ Edition, v. 22, 497. (1997)

4. Wen, J.Y. and Wilkes, G.L. - Organic/inorganic hybrid network materials by the sol-gel approach. Chem. Mat., ), 1667-1681. (1996)

5. Oréfice, R. L.; Arnold, J.; Zamora, M.; Miller, T., Brennan, A. B. - Designed Composite Interfaces using Novel Polymeric Coupling Agents. Polymer Preprints, v. 38, no 2, p. 1578. (1997)

6. Hench, L. L. - Biochemical processing of materials: a review. In: Better ceramics through chemistry VI. Ed. Materials Research Society, Pittsburgh, PA, 993-1004. (1994) 